\title{
Biobased Chemicals: 1,2,4-Benzenetriol, Selective Deuteration and Dimerization to Bifunctional Aromatic Compounds
}

\author{
Caelan Randolph, ${ }^{\dagger}$ Ciaran W. Lahive, ${ }^{\dagger}$ Selim Sami, ${ }^{\ddagger}, \S \odot$ Remco W. A. Havenith, ${ }^{\ddagger}, \S^{\prime} \|_{\odot}$ Hero J. Heeres, ${ }^{\dagger}$. \\ and Peter J. Deuss* ${ }^{\dagger} \dagger$ \\ ${ }^{\dagger}$ Department of Chemical Engineering, ENTEG, University of Groningen, Nijenborgh 4, 9747 AG Groningen, The Netherlands \\ ${ }^{*}$ Theoretical Chemistry, Zernike Institute for Advanced Materials and Stratingh Institute for Chemistry, University of Groningen, \\ Nijenborgh 4, 9747 AG Groningen, The Netherlands \\ ${ }^{\S}$ Stratingh Institute for Chemistry, University of Groningen, Nijenborgh 4, 9747 AG Groningen, The Netherlands \\ "Ghent Quantum Chemistry Group, Department of Inorganic and Physical Chemistry, Ghent University, Krijgslaan 281 (S3), \\ B-9000 Gent, Belgium
}

\section{Supporting Information}

\begin{abstract}
Benzenetriol (BTO), sourced from the carbohydrate-derived platform chemical 5-hydroxylmethylfurfural (HMF), is an interesting starting point for the synthesis of various biobased aromatic products. However, BTO readily undergoes dimerization and other reactions under mild conditions, making analysis and isolation challenging. To both control and utilize the reactivity of BTO to produce biobased building blocks, its reactivity needs to be better understood. Here it was found that specific BTO aromatic $\mathrm{C}-\mathrm{H}$ bonds are reactive toward deuterium exchange with $\mathrm{D}_{2} \mathrm{O}$, which appears pronounced under acidic conditions at room temperature and can lead to the selective formation of BTO with an aromatic ring that contains one or two deuterium atoms, the first at the five and the second at the three position. By exposure to air, it was shown that BTO forms a 5,5'-linked BTO dimer [1,1'-biphenyl $]-2,2^{\prime}, 4,4^{\prime}, 5,5^{\prime}$-hexaol (1) and subsequently a hydroxyquinone containing dimeric structure $2^{\prime}, 4,4^{\prime}, 5^{\prime}$-tetrahydroxy-[1,1'-biphenyl]-2,5-dione (2). Additionally, condensed dimer dibenzo[b, $\left.d\right]$ furan-2,3,7,8-tetraol (3) can be relatively easily accessed. The controlled formation of these symmetric and asymmetric multifunctional dimers illustrates diverse possibilities for BTO to be converted to valuable biobased aromatic compounds. Deuterium exchange was attributed to electrophilic aromatic substitution because this reactivity was found to be independent of oxygen and acid mediated. On the contrary, the dimerization was dependent on the presence of oxygen and thus likely involves radical intermediates. Thus this report overall displays different accessible reaction pathways for BTO that can be exploited for the production of BTO-derived compounds.
\end{abstract}

KEYWORDS: biobased chemicals, dimerization, hydroxybenzenes, deuteration, hydroxyquinone

\section{INTRODUCTION}

Many valuable chemicals are derived from finite fossil resources, and thus useful renewable alternatives are needed to create a sustainable chemical industry. Therefore, the production of renewable chemicals from lignocellulosic biomass such as wood and crop residues is of major interest. ${ }^{1}$ A significant role has been allocated to 5-hydroxymethylfurfural (HMF), a renewable platform chemical that can be obtained in good yields from carbohydrates and can be converted into many interesting chemicals, like levulinic acid, adipic acid, 1,6-hexanediol, caprolactam, caprolactone, and a number of furanic compounds. ${ }^{2}$ The production of C6aromatic compounds from HMF is also of high interest. ${ }^{3}$ Recently, we have shown that HMF under aqueous (sub)supercritical conditions in the presence of Lewis acidic metal salts can be converted to 1,2,4-benzenetriol (BTO, Scheme 1). ${ }^{4}$ Historically, BTO was identified as an undesired side product in many reactions that involve HMF as a product or starting material, especially under hydrothermal conditions. ${ }^{5}$ However, there is only a limited number of reports that target the production of BTO from HMF., ${ }^{4,6}$ This is also partly due to its reported instability, hindering adequate product analysis. ${ }^{5 a, c}$
Our initial findings showed that the main reaction pathway for BTO is symmetric dimerization to give $\left[1,1^{\prime}\right.$-biphenyl $]$ $2,2^{\prime}, 4,4^{\prime}, 5,5^{\prime}$-hexaol (1), which occurs readily at room temperature when exposed to air (Scheme 1). ${ }^{4}$

In addition to the formation of $\mathbf{1}$, we here report the further oxidation of 1 to a hydroxyquinone-containing dimer $2^{\prime}, 4,4^{\prime}, 5^{\prime}$-tetrahydroxy-[1,1'-biphenyl]-2,5-dione (2, Scheme $1)$. Also discussed is the synthesis of the condensed dibenzo[ $b, d]$ furan-2,3,7,8-tetraol dimer (3), which is a rigid symmetric dimer of BTO. In particular, the symmetric BTO dimers are interesting because these provide a canvas for symmetric modification and difunctionalization to obtain bifunctional molecules with potential use as building blocks for polymeric products. Additionally, during these studies, the selective deuteration of the aromatic $\mathrm{C}-\mathrm{H}$ bonds of $\mathrm{BTO}$ with $\mathrm{D}_{2} \mathrm{O}$ at room temperature was explored, which was readily

Special Issue: Work from the Organic Reactions Catalysis Society Meeting 2018

Received: September 14, 2018

Published: November 9, 2018 
Scheme 1. Overview of the Previously Reported Formation of BTO ${ }^{4}$ and Subsequent BTO Chemistry Discussed in This Work

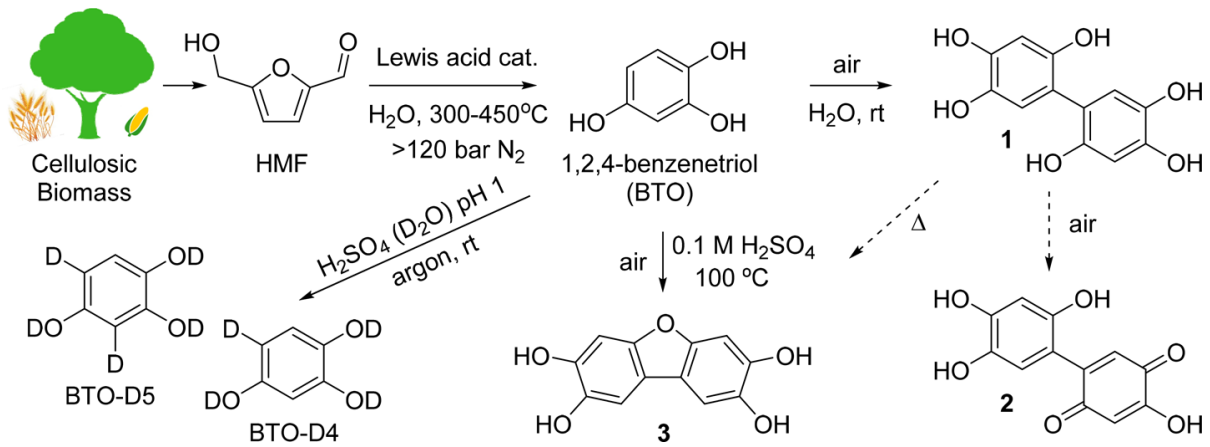

achieved under acidic conditions to obtain BTO-D4 and BTOD5.

\section{RESULTS AND DISCUSSION}

Dimer $\mathbf{1}$ is formed from BTO under aerobic conditions in water (Scheme 1). ${ }^{4}$ A crystal structure was obtained, confirming the $\mathrm{C}-\mathrm{C}$ bridged structure on the five position of both BTO molecules involved in the reaction and confirming previously suggested structures. ${ }^{7}$ Under mild conditions the formation of $\mathbf{1}$ is relatively slow, but because the product tends to precipitate or crystallize from water, this can hamper the accurate analysis of solutions containing BTO. ${ }^{4,5 \mathrm{c}}$ The formation of $\mathbf{1}$ and other dimers and oligomers is associated with significant darkening of the clear BTO solution, as illustrated by the UV-vis spectra of the isolated compounds (Figure S1). For the purpose of controlling the reaction of BTO to 1, we aimed to study its reactivity under varying conditions to either prevent or promote its formation.

Initial studies on the dimerization of BTO were performed under a range of conditions (acid, base, air, light) in $\mathrm{D}_{2} \mathrm{O}$ by following the reactions in time using nuclear magnetic resonance (NMR) with an internal standard (Figure 1a). From these experiments, the effect of temperature and the addition of acids and base was evident. All of these were found to increase the rate of BTO conversion significantly compared with BTO conversion at room temperature and neutral $\mathrm{pH}$. Analysis by mass spectroscopy $\left(\mathrm{ES}^{+}\right)$showed that under the acidic conditions and increased temperature, $\mathbf{1}$ is the major product, whereas basic conditions promoted the formation of a range of products including $\mathbf{1}$, trimers, and possibly higher oligomers (Figure S2).

While following these reactions in $\mathrm{D}_{2} \mathrm{O}$ by $\mathrm{NMR}$, an unexpected phenomenon was observed that did affect the analysis of BTO conversion to its dimer $\mathbf{1}$, namely, deuterium exchange of BTO's aromatic $\mathrm{C}-\mathrm{H}$ bonds to generate BTO-D4 and BTO-D5 (Scheme 2). This is in addition to the expected exchange at the $\mathrm{Ar}-\mathrm{OH}$ signals, which instantly generates BTO-D3. This could clearly be observed by tracking the ratios of the $\mathrm{Ar}-\mathrm{H}$ signals that started fluctuating in time even before the significant formation of dimer 1 . This is represented in Figure $1 \mathrm{~b}$ by the change in intensity of the different signals of BTO, as shown by NMR monitoring over an extended time period. When the integrals of the $\mathrm{Ar}-\mathrm{H}$ signals are individually monitored against the internal standard, Figure $1 \mathrm{c}$ is obtained, showing the different rates of signal loss associated with deuteration for BTO. The signal for the 5-Ar-H $(\delta 6.40, \mathrm{dd}$, ${ }^{3} \mathrm{~J}_{\mathrm{HH}}=8.7 \mathrm{~Hz},{ }^{4} \mathrm{~J}_{\mathrm{HH}}=2.8 \mathrm{~Hz}$ ) decreased relatively rapidly under acidic conditions, already seeing a significant decrease after $1 \mathrm{~h}$. By the time the subsequent sample was taken, after $70 \mathrm{~h}$, the signal 5-Ar-H had already almost completely disappeared, transforming the doublets for the protons at the six and three positions $\left(\delta 6.84, \mathrm{~d},{ }^{3} \mathrm{~J}_{\mathrm{HH}}=8.7 \mathrm{~Hz}\right.$ and $\delta 6.53$, ${ }^{4} \mathrm{~J}_{\mathrm{HH}}=2.8 \mathrm{~Hz}$, respectively) to singlets by loss of coupling to the neighboring hydrogen. These singlets were also relatively broad due to $\mathrm{H}-\mathrm{D}$ coupling. This indicated that in this stage BTO-D4 was nearly exclusively present in solution. The singlet for the 3-Ar-H of BTO decreased compared with that for the 6-Ar-H over time. After $500 \mathrm{~h}$, this signal had also completely disappeared, showing the selective formation of BTO-D5. The signal of 6-Ar-H also weakened in time compared with the internal standard; however, this is more likely associated with the formation of $\mathbf{1}$, which could be shown by an increase in the respective signal for $\mathrm{H} 6$ and $\mathrm{H}^{\prime}(\delta 6.80, \mathrm{~s})$. The deuterium exchange was also visible for the 3 and $3^{\prime}$ positions of 1 ( $\delta$ $6.62, \mathrm{~s})$, with a clear decrease in this signal in time compared with the H6-6' signal. The deuteration of the three and five positions of BTO could also be observed when a ${ }^{2} \mathrm{H}$ NMR spectrum was recorded (Figure S3). In a separate experiment, it was determined that the deuterium exchange at the five position is actually completed after $24 \mathrm{~h}$ and is thus relatively fast (Figure 1e,f). This reaction is remarkable because it involves selective deuterium exchange of aromatic $\mathrm{C}-\mathrm{H}$ bonds at room temperature in acidic $\mathrm{D}_{2} \mathrm{O}$ and was even observed at neutral $\mathrm{pH}$ in $\mathrm{D}_{2} \mathrm{O}$. Such reactions are typically only reported under more forcing conditions by, for example, the application of strong bases and acids, elevated temperatures, or transitionmetal catalysts. ${ }^{8}$

Further experiments to investigate BTO reactivity were conducted in nondeuterated water to avoid deuterium exchange effects aiming to specifically investigate the formation of dimer $\mathbf{1}$ (Scheme 3). This compound is clearly identified in the ${ }^{1} \mathrm{H}$ NMR spectrum in DMSO- $d_{6}$ by the proton signals of the 3-Ar-H and 6-Ar-H at $\delta 6.33$ and $\delta 6.51$, respectively, and is already present in small amounts in most commercial samples of BTO (SI Section 1). To demonstrate the stability of BTO solutions upon the exclusion of oxygen, an experiment was set up on a $400 \mathrm{mg}$ scale and monitored in time while alongside comparable experiments were performed where the solution was left to stand exposed to air with and without the exclusion of light. Samples were taken and subsequently analyzed by NMR over a time period of $5000 \mathrm{~h}$ by the removal of the solvent from the collected aliquot and the solvation of the dried material in DMSO- $d_{6}$. BTO and $\mathbf{1}$ were quantified using the average integration of all $\mathrm{Ar}-\mathrm{H}$ signals against dimethyl sulfone, which was used as an internal standard (Figure 2). The reaction in the absence of air did indeed show that BTO is completely stable for at least 5 months when stored under the 

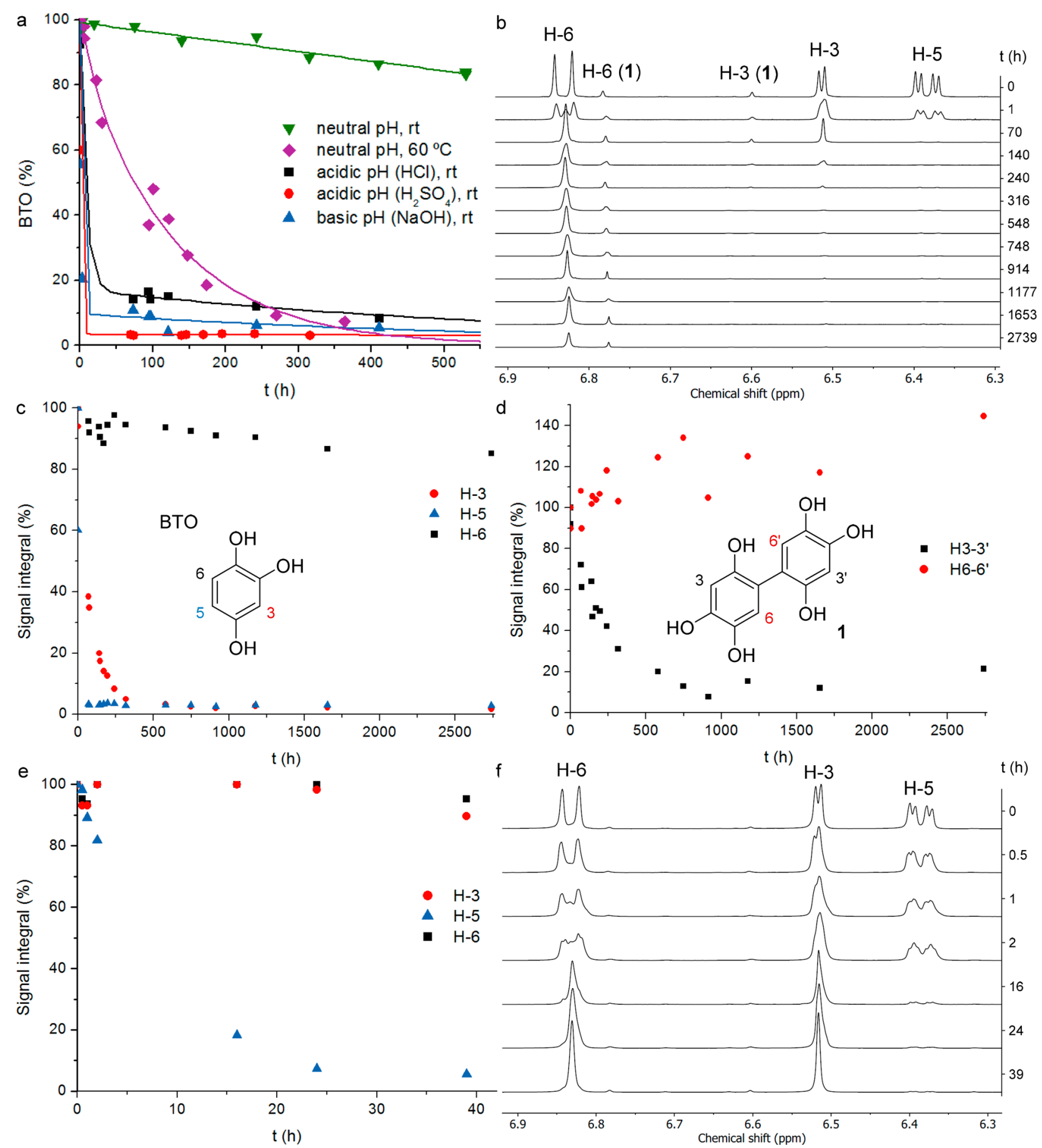

Figure 1. (a) Decomposition of BTO (TCI) in $\mathrm{D}_{2} \mathrm{O}(0.125 \mathrm{M})$ as a function of time under different conditions (acidic: $\mathrm{pH} 1$, basic: $\left.\mathrm{pH} 10\right)$ monitored by ${ }^{1} \mathrm{H}$ NMR via the signal of the 5-Ar-H compared with an internal standard. (For the illustration, lines are added, obtained through fitting a second-order exponential decay.) (b) Series of ${ }^{1} \mathrm{H}$ NMR spectra of BTO in acidic $\left(\mathrm{H}_{2} \mathrm{SO}_{4}\right) \mathrm{D}_{2} \mathrm{O}$. Relative decrease in different signal integrals compared with integrals at $t_{0}$ for different aromatic protons of (c) BTO and (d) 1. (e) Repeat of spectra series of BTO (Aldrich) in acid $\left(\mathrm{H}_{2} \mathrm{SO}_{4}\right)$ and $\mathrm{D}_{2} \mathrm{O}$ on a short time scale. (f) Relative decrease in different signal integrals compared with integrals at $t_{0}$ for different aromatic protons of $\mathrm{BTO}$ on a shorter time scale.

\section{Scheme 2. Selective Sequential Deuteration of BTO}

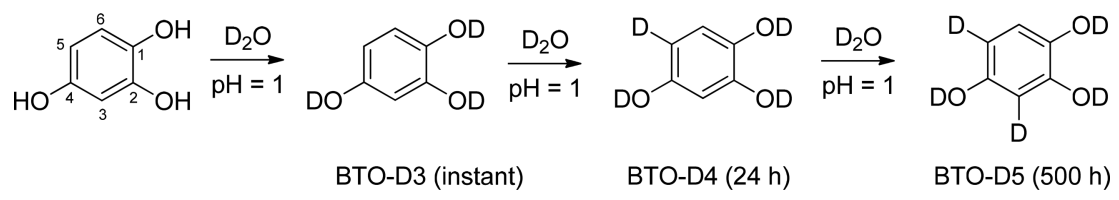


Scheme 3. Formation of the 5-5'-Linked BTO Dimer 1 upon Exposure to Air

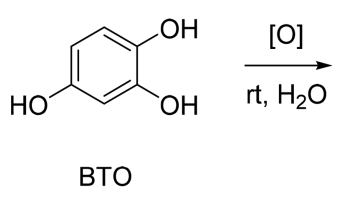<smiles>Oc1cc(O)c(-c2cc(O)c(O)cc2O)cc1O</smiles><smiles>O=C1C=C(c2cc(O)c(O)cc2O)C(=O)C=C1O</smiles>

exclusion of air (Figure 2a, Figure S5). No further formation of the 5,5-dimer 1 was observed apart from the 5,5'-dimer 1 that already appeared to be present in the commercial sample used in this study (Figure S10). When a solution with the same concentration of BTO was exposed to air and monitored, a completely different picture arose (Figures $2 \mathrm{~b}$ and 3 ). Complete conversion of BTO is observed after nearly 3 months. Here BTO is converted into its $5-5^{\prime}$-linked dimer $\mathbf{1}$. A similar reactivity pattern was observed for BTO, which was excluded from light and left to stand in air (Figure 2c, Figure S6). This demonstrated that the exposure to air is the sole requirement for the conversion of BTO at room temperature, and only protection from light is not effective for longer storage of BTO solutions.

The dimerization of BTO was shown not to be completely selective toward 1. Interestingly, at long exposure to air (1000+ $\mathrm{h})$, an additional set of signals appeared in the ${ }^{1} \mathrm{H}$ NMR (signals C in Figure 3), which did not correlate to oligomeric BTO structures previously observed under stronger oxidative or basic conditions. ${ }^{4}$ These signals were assigned to a hydroxyquinone-containing dimeric structure 2 (Scheme 3 ). On the basis of the reaction profile, 2 seemed to form from 1 over time. The structural characterization was performed by NMR analysis of $\mathbf{2}$ in a mixture containing 1 (Figures S16S18). The ${ }^{1} \mathrm{H}$ NMR showed four singlets in and the absence of J-coupling partners, which indicates hydrogens in para positions. The ${ }^{13} \mathrm{C}$ NMR displayed 12 carbon signals, including two in the region above $\delta 180$, indicating carbonyls. Overall, the number of signals in ${ }^{1} \mathrm{H}$ NMR and ${ }^{13} \mathrm{C}$ NMR suggested an asymmetric structural version of 1. HMBC-NMR (Figure 4) analysis on a sample obtained after $5982 \mathrm{~h}$ helped to verify the hydroquinone-dimer structure of $\mathbf{2}$ by showing that the carbonyls are located on the same ring. The NMR signals of the hydroxyquinone part of $\mathbf{2}$ were comparable to those reported for other substituted hydroxyquinones found in literature, supporting the proposed structure. ${ }^{9}$ The remaining set of signals was similar to that of $\mathbf{1}$. The formation of $\mathbf{2}$ was suggested in literature from the 1960 s but never confirmed. ${ }^{10}$ Oxidation leading to other (hydroxy)quinones has been reported in the literature for related structures, including BTO. ${ }^{7,11}$ Additionally, hydroxyquinones are known intermediates in the biodegradation of phenolic compounds. ${ }^{12}$

The formation of the dimeric structure 1 from BTO by exposure to oxygen was initially identified as an undesired degradation pathway that can hamper accurate analysis. Nevertheless, because of the symmetric functionalized nature of $\mathbf{1}$, it can also be viewed as an interesting starting material for polymeric materials and other applications. As previously discussed, the formation of $\mathbf{1}$ was slow at room temperature, taking 2000 $+\mathrm{h}$ under neutral conditions to reach full conversion, as evidenced by Figure 3. Therefore, we investigated more convenient conditions for the synthesis of 1. It was already shown in the first section that increasing the temperature and the addition of acid and bases did cause significant BTO conversion, although this was partly obscured by deuterium exchange. Basic conditions afforded a range of side products, including oligomeric structures, and were thus deemed unsuitable. Different conditions using acids and increased temperature (up to $100{ }^{\circ} \mathrm{C}$ ) significantly increased BTO conversion up to $70 \%$ in $20 \mathrm{~h}$ (Table S1). However, these reactions gave inconsistent results due to the partial precipitation of products from the solution in different stages of the reaction, causing the formation of undesired side products. Higher conversion of BTO to 1 could be achieved by performing the reaction without solvent. It was found that melting BTO $\left(\mathrm{mp}=140.5{ }^{\circ} \mathrm{C}\right)^{13}$ under aerobic conditions resulted in the rapid formation of dimer 1 in short reaction times $(2 \mathrm{~h})$. The obtained solid contained 1 in $80 \%$ purity, with some unreacted BTO, which could readily be removed by subsequent washings with cold water. This fast synthesis technique is detailed in SI Section 7. In general, it was found that although BTO conversion could be improved by prolonged reaction times or the application of harsher conditions, this was at the expense of BTO to dimer $\mathbf{1}$ selectivity due to the formation of side products like 2, 3 (see below), and other oligomers. Low selectivity was highly undesirable because dimer 1 was difficult to separate from such side products. However, the separation of $\mathbf{1}$ from BTO was straightforward, and thus running reactions at lower conversion with high selectivity was preferred. A mixture of BTO and 1 could be separated by the addition of cold water and centrifugation to settle the dimer. This technique allowed one to obtain pure samples of $\mathbf{1}$ from mixtures of BTO and $\mathbf{1}$. Nevertheless, because of low conversion, only $48 \%$ yield could be achieved in water, with $44 \%$ isolated yield of 1 ( $92 \%$ purity, NMR). Subsequently, 1 can be crystallized from a solution containing leftover BTO; however, yields are low to moderate.

One interesting side product that was encountered from the attempts to form $\mathbf{1}$ in water was condensed dimer 3 (Scheme 4, Figures S19-S22 for NMR spectra). This product was initially identified from an attempt to purify 1 by sublimation under high vacuum, in which small amounts of a pure condensed solid were identified as 3 (Figure S8). Thus 3 can be formed from 1 by expelling water, as was suggested more than a century ago ${ }^{14}$ but never confirmed by more advanced analytical techniques, such as NMR. Like 1, $\mathbf{3}$ is obtained as a black solid and has significant absorption in the visible region in aqueous solution (Figure S1). 3 can be directly accessed from BTO by refluxing BTO in an acidic aqueous solution at elongated reactions times. By boiling a solution of BTO $(0.2$ $\mathrm{M})$ in an aqueous $\mathrm{H}_{2} \mathrm{SO}_{4}$ solution $(0.1 \mathrm{M})$ for $60 \mathrm{~h}$ while exposed to air, near-quantitative yield of 3 was obtained (Scheme 4). 3 could subsequently be obtained as a dark-brown solid by solvent extraction. Refluxing the solution proved to be important, as the reaction at $95{ }^{\circ} \mathrm{C}$ showed only $8 \%$ yield of 3 after $65 \mathrm{~h}$ at $34 \%$ conversion of BTO, with the remainder being dimer $\mathbf{1}$ and other unidentified products. Interestingly, $\mathrm{HCl}$ was shown to be completely ineffective for the formation of $\mathbf{3}$, as only trace amounts were detected when the reaction was performed with this acid instead of $\mathrm{H}_{2} \mathrm{SO}_{4}$. In summary, it was 

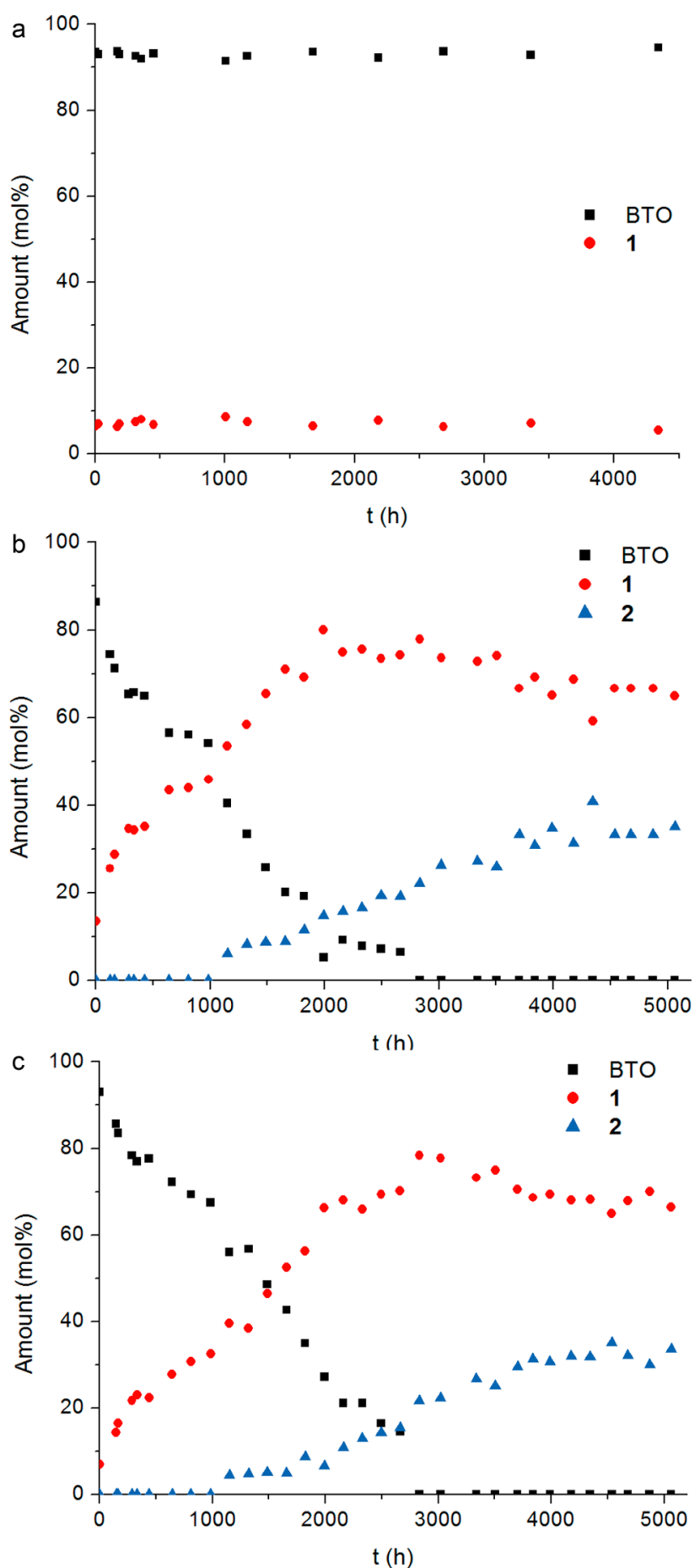

Figure 2. Aqueous BTO stability and dimer formation in time. (a) Under exclusion of air. (b) Open to air. (c) Open to air with exclusion of light ( $160 \mathrm{mM}$ initial BTO concentration, monitored by ${ }^{1} \mathrm{H}$ NMR by using dimethyl sulfone as internal standard). Note: After the levels of 1 became relatively high $(>40 \%)$, precipitate could be clearly observed. Before sampling, some effort was taken to solubilize this precipitate using sonication, but the formation of some precipitate as well as different efficiencies at solubilizing the material could be the reason for the fluctuations sometimes observed in these graphs.

thus demonstrated that both dimers 1 and 3 can be readily obtained from BTO. The synthesis of $\mathbf{3}$ was found to be more straightforward compared with that of $\mathbf{1}$, which is likely due to the high resistance of $\mathbf{3}$ to further reactions to form trimers and higher oligomers. This set of reactions allows for the development of more effective methods for producing these interesting symmetric polyphenolic compounds.

Overall, two types of reactivity were found for BTO in this work, oxidation and deuterium exchange. First, BTO can be oxidized by air to form $\mathbf{1}$. Subsequently, 1 can be further oxidized to $\mathbf{2}$ by air. The first oxidation step is likely to proceed via a two-electron oxidation step with radical intermediates that are recombined to form $\mathbf{1}$ (Scheme 5a). The regioselectivity for the formation of the $5-5^{\prime}$ dimer is the result of the relative stability of radicals on the five and three positions over the six position of the aromatic ring due to the superior stabilization of having two phenolic groups in the ortho/para or ortho/ortho positions, respectively. This is combined with reduced steric hindrance at the five position compared with the three position, leading to increased reactivity for the coupling reaction. Indeed, we previously reported that creating more forcing conditions by the addition of hydrogen peroxide afforded $3-5$ coupling. ${ }^{4}$ The oxidation of 1 to 2 seems more favored compared with $3-3$ or 3-5 coupling at room temperature upon exposure to air. Also, this reaction likely proceeds by a two-electron oxidation, generating water from oxygen. No formation of hydroxyquinone was observed directly from BTO, indicating that the formation of 1 is preferred from $\mathrm{BTO}$, whereas for $\mathbf{1}$, hydroxyquinone formation is preferred over further $\mathrm{C}-\mathrm{C}$ bond formation. Second, the observed deuteration of the aromatic $\mathrm{C}-\mathrm{H}$ bonds is likely to proceed via the deuteration of the aromatic ring of BTO-D3 to D-BTO-D3 and subsequent rearomatization to BTO-D4 (Scheme 5b). Quantum-mechanical (QM) calculations revealed $\mathrm{p} K_{\mathrm{a}}$ values ${ }^{15}$ for D-BTO-D3 with conjugate bases BTO-D3 and BTO-D4 of -4.13 and -5.66 , respectively. This is in line with the reported $\mathrm{pK}_{\mathrm{a}}$ of -3.37 determined for protonated 1,3,5-benzenetriol, ${ }^{16}$ which is expected to be slightly less acidic due to the positions of the hydroxyl groups compared with 1,3,4-benzenetriol. In this deuteration pathway, the reactivity pattern of the aromatic $\mathrm{C}-\mathrm{H}$ bonds will be the same as that for electrophilic aromatic substitution. ${ }^{17}$ The observed selectivity is due to the preferred location of the carbocation. Resonance stabilization is more effective at positions two, four, and six compared with positions one, three, and five due to the positioning of the oxygen atoms. Position five is more reactive for deuteration compared with position three due to steric effects, but after longer reaction times, the latter is also deuterated via the same mechanism. Additionally, the formation of BTO-D4 is energetically preferred over BTO-D3 (Table S2) due to lower zero-point vibrational energy, which, together with the excess $\mathrm{D}_{2} \mathrm{O}$, shifts the equilibrium toward BTO-D4 and BTO-D5. In principle, the deuteration of BTO can also be explained by the radical initiation shown for the formation of $\mathbf{1}$. However, deuteration was still observed under the exclusion of air with no apparent change in reaction rates or reactivity (Figure S4).

\section{CONCLUSIONS}

Biobased 1,2,4-benzenetriol, an aromatic product attainable from 5-hydroxymethylfurfural (HMF), can be used as a polyphenolic building block. In this study, it is shown that BTO possesses interesting reactivity. Although this reactivity can provide challenges for the accurate analysis of BTO in studies where its reactivity is not carefully considered, here it was shown that this reactivity can lead to the formation of biobased compounds that can have potential as chemical intermediates. First, it was shown that previously reported 5- 


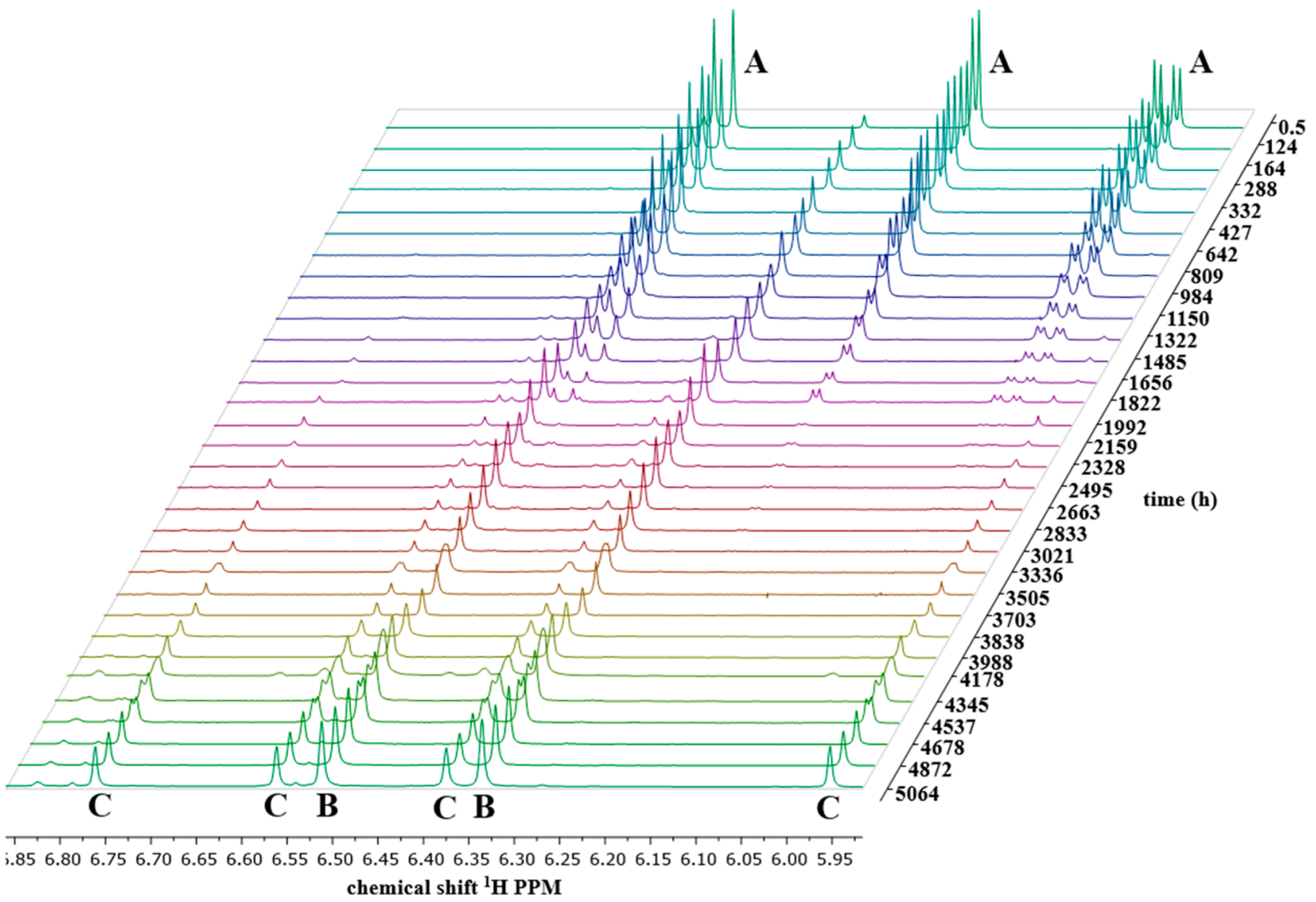

Figure 3. Series of ${ }^{1} \mathrm{H}$ NMR spectra in DMSO- $d_{6}$ obtained by sampling an aqueous BTO solution in time. (A) 1,2,4-Benzenetriol (BTO), (B) dimer $\mathbf{1}$, and (C) hydroquinone dimer 2 . This spectrum series was used to generate Figure $2 \mathrm{~b}$.

$5^{\prime}$-linked BTO dimer $\left[1,1^{\prime}\right.$-biphenyl]-2,2',4,4',5,5'-hexaol (1) is formed exclusively under aerobic conditions at room temperature, which likely proceeds via a radical intermediate. Furthermore, 1 can be subsequently oxidized to afford a hydroxyquinone-containing dimeric structure, $2^{\prime}, 4,4^{\prime}, 5^{\prime}$-tetrahydroxy-[1,1'-biphenyl]-2,5-dione (2) under the same conditions. It was also demonstrated that dimer $\mathbf{1}$, as well as a condensed dimer dibenzo[b,d]furan-2,3,7,8-tetraol (3), can be readily produced from BTO. The symmetric nature of these two dimeric products provides a potential platform for biobased polymeric products as, for example, bisphenol $\mathrm{A}^{18}$ replacements. This is one of the future directions that will be explored in our group. Additionally, it was shown that the specific sequential deuterium exchange of the aromatic $\mathrm{C}-\mathrm{H}$ bonds of BTO is readily achieved under acidic conditions at room temperature. Deuterium incorporation first occurs at the five position, followed by the three position to yield BTO with four and five deuterium atoms, respectively, which is likely to proceed through electrophilic aromatic substitution. These studies exemplify two types of reactivity that BTO can be involved in, which should be considered when working with this compound and which can also be exploited for accessing biobased compounds from BTO.

\section{EXPERIMENTAL SECTION}

Sequential BTO Deuteration in Acidic $\mathrm{D}_{2} \mathrm{O}$. An NMR tube was charged with BTO $(10 \mathrm{mg}, 0.08 \mathrm{mmol})$ and DMSO as internal standard ( $4 \mathrm{mg}, 0.05 \mathrm{mmol})$. A measured amount of $\mathrm{D}_{2} \mathrm{O}(0.5 \mathrm{~mL})$ was added as solvent. $\mathrm{D}_{2} \mathrm{O}$ was acidified to $\mathrm{pH}$
1 using concentrated $\mathrm{H}_{2} \mathrm{SO}_{4}$ (nondeuterated). The reaction was kept at the desired temperature. NMR spectra were collected at defined time points, and the signal intensities of the BTO protons were determined by integration relative to the signal of the internal standard. ${ }^{1} \mathrm{H}$ NMR $\delta(400 \mathrm{MHz}$, $\left.\mathrm{D}_{2} \mathrm{O}\right)$ : BTO: $6.84\left(\mathrm{~d}, 1 \mathrm{H},{ }^{3} \mathrm{~J}_{\mathrm{HH}}=8.7 \mathrm{~Hz}, \mathrm{C} 6-\underline{\mathrm{H}}\right), 6.53(\mathrm{~d}, 1 \mathrm{H}$, $\left.{ }^{4} \mathrm{~J}_{\mathrm{HH}}=2.8 \mathrm{~Hz}, \mathrm{C} 3-\underline{\mathrm{H}}\right), 6.40\left(\mathrm{dd}, 1 \mathrm{H},{ }^{4} \mathrm{~J}_{\mathrm{HH}}=2.8, \mathrm{~Hz},{ }^{3} \mathrm{~J}_{\mathrm{HH}}=8.7\right.$ $\mathrm{Hz}, \mathrm{C} 5-\underline{\mathrm{H}})$.

BTO Stability in $\mathrm{H}_{2} \mathrm{O}$. A clarified polypropylene centrifuge tube $(50 \mathrm{~mL})$ was charged with BTO $(400 \mathrm{mg}, 3.2 \mathrm{mmol})$ and $\mathrm{H}_{2} \mathrm{O}(20 \mathrm{~mL})$. For the experiment with the exclusion of air, the $\mathrm{H}_{2} \mathrm{O}$ was degassed by repeated freeze-pump-thaws under an argon atmosphere, and the contents were added under an argon atmosphere. For the experiment with exclusion of light, the tube was wrapped in aluminum foil. To sample each solution, an aliquot was abstracted from each reaction vessel, weighed (about 0.2 to $0.4 \mathrm{~g}$ ), and placed in a centrifuge tube $(1.5 \mathrm{~mL})$. The sample was frozen in liquid nitrogen and freezedried to remove all moisture. Dimethyl sulfone of known quaintly was added to each centrifuge tube containing dry substrate as an internal standard, and DMSO- $d_{6}$ was used to solvate all contents. The DMSO- $d_{6}$ solution was transferred to an NMR tube, and quantitative ${ }^{1} \mathrm{H}$ NMR was recorded (32 scans, $2 \mathrm{~s}$ delay). The solution constituents were quantified by integration against internal standard dimethyl sulfone using the average of integration for the signals corresponding to the aromatic $\mathrm{C}-\mathrm{H}$ bonds. For the experiment run under the exclusion of air, all of these steps were performed under an argon atmosphere. Because of the water insolubility of the 


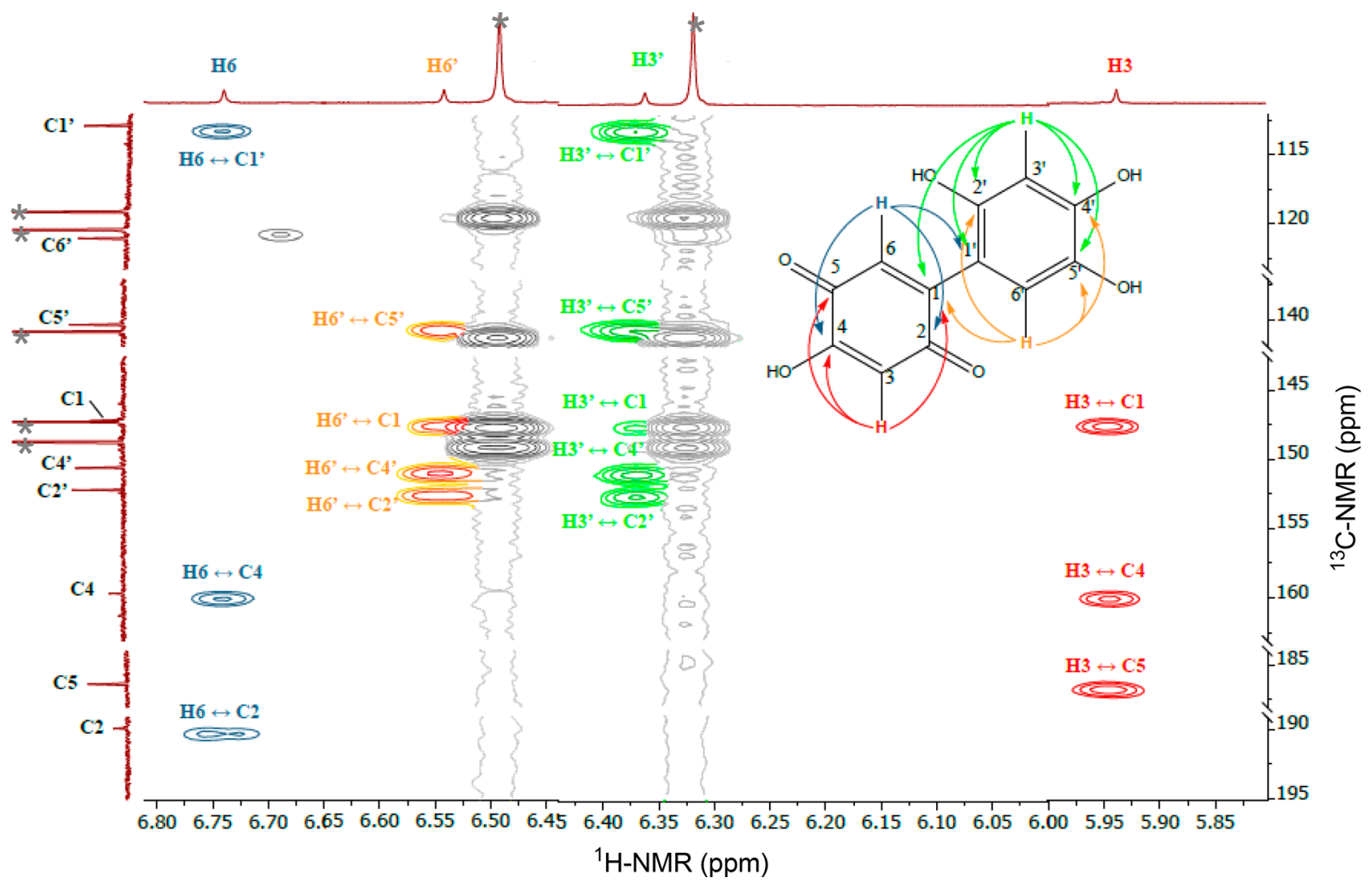

Figure 4. Selected area of the HMBC-NMR spectrum in DMSO- $d_{6}$ of a sample (after $5982 \mathrm{~h}$ ) containing the hydroxyquinone motif containing dimer 2. (Gray signals labeled with * belong to the 5,5'-BTO dimer 1.)

Scheme 4. Synthesis of 3 from BTO<smiles></smiles>

Scheme 5. Proposed Mechanism for the (a) Dimerization and (b) Deuterium Exchange Reaction Observed for BTO in This Work<smiles>Oc1ccc(O)c(O)c1</smiles><smiles></smiles>

dimeric products, extensive shaking or sonication was necessary before each sampling. Observed NMR signals for the different quantified species: BTO: ${ }^{1} \mathrm{H}$ NMR $\delta(400 \mathrm{MHz}$, DMSO- $\left.d_{6}\right): 8.65(\mathrm{~s}, 1 \mathrm{H}, \mathrm{C} 4-\mathrm{OH}), 8.47(\mathrm{~s}, 1 \mathrm{H}, \mathrm{C} 2-\mathrm{OH})$, $8.01(\mathrm{~s}, 1 \mathrm{H}, \mathrm{C} 1-\mathrm{O} \underline{\mathrm{H}}), 6.48\left(\mathrm{~d}, 1 \mathrm{H},{ }^{3} \mathrm{~J}_{\mathrm{HH}}=8.5 \mathrm{~Hz}, \mathrm{C} 6-\underline{\mathrm{H}}\right)$, $6.20\left(\mathrm{~d}, 1 \mathrm{H},{ }^{4} \mathrm{~J}_{\mathrm{HH}}=2.8 \mathrm{~Hz}, \mathrm{C} 3-\underline{\mathrm{H}}\right), 6.00\left(\mathrm{dd}, 1 \mathrm{H},{ }^{4} \mathrm{~J}_{\mathrm{HH}}=2.8\right.$, $\left.\mathrm{Hz},{ }^{3} \mathrm{~J}_{\mathrm{HH}}=8.5 \mathrm{~Hz}, \mathrm{C} 5-\underline{\mathrm{H}}\right) .{ }^{13} \mathrm{C}$ NMR $\delta\left(100 \mathrm{MHz}, \mathrm{DMSO}-d_{6}\right)$ : 150.2 (C4), 145.7 (C2), 137.6 (C1), 115.8 (C6), 105.1 (C5), 103.5 (C3). 1: ${ }^{1} \mathrm{H}$ NMR $\delta\left(400 \mathrm{MHz}, \mathrm{DMSO}-d_{6}\right): 8.71(\mathrm{~s}, 2 \mathrm{H}$, $\left.\mathrm{C} 4,4^{\prime}-\mathrm{OH}\right), 8.50$ (s, 2H, C2,2'-OH ), 8.20 (s, $\left.2 \mathrm{H}, \mathrm{C} 1,1^{\prime}-\mathrm{OH}\right)$,

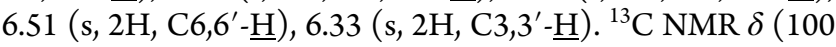
$\left.\mathrm{MHz}, \mathrm{DMSO}-d_{6}\right): 146.4\left(\mathrm{C} 4,4^{\prime}\right), 145.0 \quad\left(\mathrm{C} 2,2^{\prime}\right), 138.5$ $\left(\mathrm{C} 1,1^{\prime}\right), 118.1\left(\mathrm{C} 6,6^{\prime}\right), 116.8\left(\mathrm{C} 5,5^{\prime}\right), 104.6\left(\mathrm{C} 3,3^{\prime}\right) . \mathrm{T}-$ FTMS - p ESI $(m / z)$ : calculated for $\mathrm{C}_{12} \mathrm{H}_{10} \mathrm{O}_{6}\left[\mathrm{M}-\mathrm{H}^{+}\right]$: 249.0394; found: 249.0398. 2: ${ }^{1} \mathrm{H}$ NMR $\delta$ (400 MHz, DMSO$\left.d_{6}\right): 11.3$ (s, Ar-OH $), 9.30$ (s, Ar-OH $), 9.10$ (s, Ar-OH $)$, 8.36 (s, Ar-O $\underline{H}), 6.75$ (s, C6- $\underline{\mathrm{H}}), 6.56$ (s, C6 $\left.6^{\prime}-\underline{\mathrm{H}}\right), 6.38$ (s, C3 $\left.{ }^{\prime}-\underline{\mathrm{H}}\right), 5.95(\mathrm{~s}, \mathrm{C} 3-\underline{\mathrm{H}}) .{ }^{13} \mathrm{C}$ NMR $\delta\left(100 \mathrm{MHz}, \mathrm{DMSO}-d_{6}\right)$ : 187.2 (C2), 183.7 (C5), 157.0 (C4), 149.5 (C2'), 148.0 (C4'), 144.8 (C1), 137.7 (C5'), 130.0 (C3), $118.4\left(\mathrm{C6}^{\prime}\right)$, $110.3\left(\mathrm{Cl}^{\prime}\right), 108.9$ (C6), $103.8\left(\mathrm{C}^{\prime}\right)$. T-FTMS - p ESI $(\mathrm{m} /$ $z)$ : calculated for $\mathrm{C}_{12} \mathrm{H}_{8} \mathrm{O}_{6}\left[\mathrm{M}-\mathrm{H}^{+}\right]$: 247.0231; found: 247.0245 .

Typical Synthesis and Purification of 1 by Reflux in $\mathrm{H}_{2}$ O. BTO (149 mg, $1.18 \mathrm{mmol}$ ) and $\mathrm{H}_{2} \mathrm{O}(6.9 \mathrm{~mL})$ were weighed in a $50 \mathrm{~mL}$ round-bottomed flask fitted with a straight tube condenser. BTO appeared as an off-white beige powder and in aqueous media afforded a golden solution. The solution was stirred and heated to reflux in a sand bath (set to $110^{\circ} \mathrm{C}$ ) for $20 \mathrm{~h}$. The solution, which appeared black with black precipitate and appeared to stain the glass, was left to cool to room temperature and subsequently freeze-dried. The dry material collected was brown in color. This material was sampled for ${ }^{1} \mathrm{H}$ NMR analysis in DMSO- $d_{6}$. Products were identified and compared via integral analysis to determine the product composition. Analysis showed a 52.9\% BTO, 43.7\% 1, 
and $3.4 \% 3$ yield. By washing the solid with cold water, dimer 1 could be isolated with $92 \%$ purity. Further purification could be achieved by crystallization from water described previously, ${ }^{4}$ but only in low yield. For analysis data, see above.

Typical Synthetic Procedure for 3. BTO (521 mg, 4.13 $\mathrm{mmol}), \mathrm{H}_{2} \mathrm{SO}_{4}(56.5 \mathrm{mg}, 0.58 \mathrm{mmol})$, and $\mathrm{H}_{2} \mathrm{O}(21 \mathrm{~mL})$ were mixed in a $50 \mathrm{~mL}$ round-bottomed flask fitted with a straight tube condenser. The solution was stirred and refluxed in a sand bath (set to $110^{\circ} \mathrm{C}$ ) for $91 \mathrm{~h}$. The obtained black solution with visible precipitate on the flask surface was left to cool to room temperature (NMR in this stage shows $>90 \% 3$ ). Tetrahydrofuran (THF) was added to dissolve all dimeric and monomer compounds and phase-separated by the addition of $\mathrm{NaCl}$ to obtain a saturated aqueous phase. The organic layer was collected, and THF was removed in vacuo. The obtained product was freeze-dried, yielding $317.0 \mathrm{mg}$ (1.36 mmol, 66\%) of isolated 3 as a brown powder. Dimer 3: ${ }^{1} \mathrm{H}$ NMR $\delta(400$ $\left.\mathrm{MHz}, \mathrm{DMSO}-d_{6}\right): 9.15$ (s, $\left.2 \mathrm{H}, \mathrm{H} 4,4^{\prime}\right), 8.76$ (s, 2H, H3,3'), $7.14\left(\mathrm{~s}, 2 \mathrm{H}, \mathrm{H} 2,2^{\prime}\right), 6.90$ (s, 2H, H5,5'). ${ }^{13} \mathrm{C}$ NMR $\delta(100$ $\left.\mathrm{MHz}, \mathrm{DMSO}-d_{6}\right): 149.6\left(\mathrm{C} 1,1^{\prime}\right), 144.6\left(\mathrm{C} 3,3^{\prime}\right), 141.9$ $\left(\mathrm{C} 4,4^{\prime}\right), 115.4\left(\mathrm{C} 6,6^{\prime}\right), 105.0\left(\mathrm{C} 2,2^{\prime}\right), 98.4\left(\mathrm{C} 5,5^{\prime}\right)$. TFTMS - p ESI $(m / z)$ : calculated for $\mathrm{C}_{12} \mathrm{H}_{8} \mathrm{O}_{5}\left[\mathrm{M}-\mathrm{H}^{+}\right]$: 231.0299; found: 231.0298 .

\section{ASSOCIATED CONTENT}

\section{S Supporting Information}

The Supporting Information is available free of charge on the ACS Publications website at DOI: 10.1021/acs.oprd.8b00303.

General information on materials and methods, UV-vis data for BTO, 1, and 3, mass spectroscopy data, additional data on the deuterium exchange, all ${ }^{1} \mathrm{H}$ NMR data for BTO stability in time, dimer 1 synthesis in water and without solvent, decomposition of $\mathbf{1}$ and formation of 3, details of the QM calculations to obtain the theoretical $\mathrm{p} K_{\mathrm{a}}$ values, and NMR spectra for BTO from different supplies, 1, 2, and 3. (PDF)

\section{AUTHOR INFORMATION}

\section{Corresponding Author}

*E-mail: p.j.deuss@rug.nl.

\section{ORCID}

Caelan Randolph: 0000-0001-6402-8895

Selim Sami: 0000-0002-4484-0322

Remco W. A. Havenith: 0000-0003-0038-6030

Hero J. Heeres: 0000-0002-1249-543X

Peter J. Deuss: 0000-0002-2254-2500

\section{Funding}

C.W.L. acknowledges Topconsortia voor Kennis en Innovatie (TKI Biobased Economy) and Syncom for funding (BBE1712). S.S. and R.W.A.H. acknowledge the research program of the Foundation of Fundamental Research on Matter (FOM), which is part of The Netherlands Organisation for Scientific Research (NWO). This contribution is part of the FOM-focus Group 'Next Generation Organic Photovoltaics', participating in the Dutch Institute for Fundamental Energy Research (DIFFER).

\section{Notes}

The authors declare no competing financial interest.

\section{ACKNOWLEDGMENTS}

We thank Qingqing Yuan, Theodora Tiemersma Wegman, and Hans van der Velde for their technical and analytical support.

\section{REFERENCES}

(1) (a) Holladay, J. E.; White, J. F.; Bozell, J. J.; Johnson, D. In Top Value-Added Chemicals from Biomass Vol. II-Results of Screening for Potential Candidates from Biorefinery Lignin; U.S. Department of Energy (DOE) by PNNL: Richland, WA, 2007; PNNL-16983. (b) Alonso, D. M.; Hakim, S. H.; Zhou, S.; Won, W.; Hosseinaei, O.; Tao, J.; Garcia-Negron, V.; Motagamwala, A. H.; Mellmer, M. A.; Huang, K.; Houtman, C. J.; Labbé, N.; Harper, D. P.; Maravelias, C. T.; Runge, T.; Dumesic, J. A. Increasing the revenue from lignocellulosic biomass: Maximizing feedstock utilization. Sci. Adv. 2017, 3, No. e1603301. (c) Clark, J. H.; Luque, R.; Matharu, A. S. Green Chemistry, Biofuels, and Biorefinery. Annu. Rev. Chem. Biomol. Eng. 2012, 3, 183.

(2) (a) van Putten, R. J.; van der Waal, J. C.; de Jong, E.; Rasrendra, C. B.; Heeres, H. J.; de Vries, J. G. Hydroxymethylfurfural, A VersatilePlatform Chemical Made From Renewable Resources. Chem. Rev. 2013, 113, 1499. (b) Kucherov, F. A.; Romashov, L. V.; Galkin, K. I.; Ananikov, V. P. Chemical Transformations of Biomass-Derived C6-Furanic Platform Chemicals for Sustainable Energy Research, Materials Science, and Synthetic Building Blocks. ACS Sustainable Chem. Eng. 2018, 6, 8064. (c) Bozell, J. J.; Petersen, G. R. Technology development for the production of bio-based products from biorefinery carbohydrates-The US Department Energy's 'Top10' revisited. Green Chem. 2010, 12, 539.

(3) (a) Settle, A. E.; Berstis, L.; Rorrer, N. A.; Roman-Leshkóv, Y.; Beckham, G. T.; Richards, R. M.; Vardon, D. R. Heterogeneous Diels-Alder catalysis for biomass-derived aromatic compounds. Green Chem. 2017, 19, 3468. (b) Bruijnincx, P. C. A.; Weckhuysen, B. M. Shale Gas Revolution: An Opportunity for the Production of Biobased Chemicals? Angew. Chem., Int. Ed. 2013, 52, 11980. (c) Pacheco, J. J.; Davis, M. E. Synthesis of terephthalic acid via Diels-Alder reactions with ethylene and oxidized variants of 5hydroxymethylfurfural. Proc. Natl. Acad. Sci. U. S. A. 2014, 111, 8363. (d) Thiyagarajan, S.; Genuino, H. C.; van der Waal, J. C.; de Jong, E.; Weckhuysen, B. M.; van Haveren, J.; Bruijnincx, P. C. A.; van Es, D. S. A Facile Solid-Phase Route to Renewable Aromatic Chemicals from Biobased Furanics. Angew. Chem., Int. Ed. 2016, 55, 1368.

(4) Kumalaputri, A. J.; Randolph, C.; Otten, E.; Heeres, H. J.; Deuss, P. J. Lewis Acid Catalyzed Conversion of 5-Hydroxymethylfurfural to 1,2,4-Benzenetriol, an Overlooked Biobased Compound. ACS Sustainable Chem. Eng. 2018, 6, 3419.

(5) (a) Srokol, Z.; Bouche, A. G.; van Estrik, A.; Strik, R. C. J.; Maschmeyer, T.; Peters, J. A. Hydrothermal upgrading of biomass to biofuel: studies on some monosaccharide model compounds. Carbohydr. Res. 2004, 339, 1717. (b) Kimura, H.; Nakahara, M.; Matubayasi, N. Solvent Effect on Pathways and Mechanisms for DFructose Conversion to 5-Hydroxymethyl-2-furaldehyde: In Situ ${ }^{13} \mathrm{C}$ NMR Study. J. Phys. Chem. A 2013, 117, 2102. (c) Chuntanapum, A.; Matsumura, Y. Formation of Tarry Material from 5-HMF in subcritical and supercritical water. Ind. Eng. Chem. Res. 2009, 48, 9837. (d) van Zandvoort, I.; Wang, Y.; Rasrendra, C. B.; van Eck, E. R. H.; Bruijnincx, P. C. A.; Heeres, H. J.; Weckhuysen, B. M. Formation, Molecular Structure, and Morphology of Humins in Biomass Conversion: Influence of Feedstock and Processing Conditions. ChemSusChem 2013, 6, 1745.

(6) (a) Luijkx, G. C. A.; van Rantwijk, F.; van Bekkum, H. Formation of 1,2,4-benzenetriol by hydrothermal treatment of carbohydrates. Recl. Trav. Pay-Bas 1991, 110, 343. (b) Luijkx, G. C. A.; van Rantwijk, F.; van Bekkum, H. Hydrothemal formation of 1,2,4-benzenetriol from 5-hydroxymethyl-2-furaldehyde and Dfructose. Carbohydr. Res. 1993, 242, 131.

(7) Li, X.; Cubbage, J. W.; Tetzlaff, T. A.; Jenks, W. S. Photocatalytic Degradation of 4-Chlorophenol. 1. The Hydroquinone Pathway. J. Org. Chem. 1999, 64, 8509. 
(8) (a) Junk, T.; Catallo, W. J. Hydrogen isotope exchange reactions involving $\mathrm{C}-\mathrm{H}$ (D, T) bonds. Chem. Soc. Rev. 1997, 26, 401. (b) Atzrodt, J.; Derdau, V.; Fey, T.; Zimmermann, J. TheRenaissanceofH/DExchange. Angew. Chem., Int. Ed. 2007, 46, 7744. (c) Banijamali, A. R.; Charalambous, A.; van der Schyf, C. J.; Makriyannis, A. Specific deuteration of phenols and aromatic ethers using boron trifluoride and deuterium oxide. J. Labelled Compd. Radiopharm. 1987, 24, 1479. (d) Zhan, M.; Xu, R.; Tian, Y.; Jiang, H.; Zhao, L.; Xie, Y.; Chen, Y. A Simple and Cost-Effective Method for the Regioselective Deuteration of Phenols. Eur. J. Org. Chem. 2015, 2015, 3370. (e) Murai, Y.; Wang, L.; Masuda, K.; Sakihama, Y.; Hashidoko, Y.; Hatanaka, Y.; Hashimoto, M. Rapid and Controllable Hydrogen/Deuterium Exchange on Aromatic Rings of $\alpha$-Amino Acids and Peptides. Eur. J. Org. Chem. 2013, 2013, 5111.

(9) Jameson, G. N. L.; Kudryavtsev, A. B.; Linert, W. The oxidation of 6-hydroxydopamine in aqueous solution. Part 1 . The formation of three metastable quinones at low pH. J. Chem. Soc., Perkin Trans. 2 2001, 0, 557.

(10) van Dijk, H.; Ter Haar, H. E. A Polarographic Study of the Oxidation of some Polyhydroxybenzenes with Atmospheric Oxygen. Recl. Trav. Chim. Pays-Bas 1961, 80, 1207.

(11) (a) Spyroudis, S. Hydroxyquinones: Synthesis and Reactivity. Molecules 2000, 5, 1291. (b) Forrest, J.; Overell, B. G.; Petrow, V.; Stephenson, O. Some Observations on the Inhibition of the Action of Hyaluronidase on Hyaluronic Acid by Gentisic Acid and its Oxidation Products. J. Pharm. Pharmacol. 1952, 4, 231. (c) Gui, Y.; Kuwana, T. Long optical Path Length Thin-Layer Spectroelectrochemistry. Catalytic Oxidation of Hydroxyquinones by Oxygen at Platinum. Chem. Lett. 1987, 16, 231.

(12) (a) Boyd, D. R.; Sharma, N. D.; Malone, J. F.; McIntyre, P. B. A.; McRoberts, C.; Floyd, S.; Allen, C. C. R.; Gohil, A.; Coles, S. J.; Horton, P. N.; Stevenson, P. J. Toluene Dioxygenase-Catalyzed Synthesis and Reactions of cis-Diol Metabolites Derived from 2- and 3-Methoxyphenols. J. Org. Chem. 2015, 80, 3429. (b) Lam, L. K. M.; Zhang, Z.; Board, P. G.; Xun, L. Reduction of Benzoquinones to Hydroquinones via Spontaneous Reaction with Glutathione and Enzymatic Reaction by S-Glutathionyl-Hydroquinone Reductases. Biochemistry 2012, 51, 5014.

(13) Teuber, H. J.; Staiger, G. Reactions with nitrosodisulfonate. VIII. o-Benzoquinones and phenazines. Chem. Ber. 1955, 88, 802.

(14) Brezina, E. Über die Alkylierung des Oxyhydrochinons. Monatsh. Chem. 1901, 22, 590.

(15) Liptak, M. D.; Shields, G. C. Accurate pK(a) calculations for carboxylic acids using Complete Basis Set and Gaussian-n models combined with CPCM continuum solvation methods. J. Am. Chem. Soc. 2001, 123, 7314.

(16) Kresge, A. J.; Chiang, Y. Aromatic Protonation. III. Kinetic Hydrogen Isotope Effects on Acid-Catalyzed Aromatic Hydrogen Exchange in 1,3,5-Trimethoxybenzene. J. Am. Chem. Soc. 1967, 89, 4411.

(17) Giles, R.; Kim, I.; Chao, W. E.; Moore, J.; Jung, K. W. Dual Studies on a Hydrogen-Deuterium Exchange of Resorcinol and the Subsequent Kinetic Isotope Effect. J. Chem. Educ. 2014, 91, 1220.

(18) Rochester, J. R. Bisphenol A and human health: A review of the literature. Reprod. Toxicol. 2013, 42, 132. 